\title{
A Remote Measurement System for Three-dimensional Magnetic Field
}

\author{
Hengli Song \\ College of Automation \\ China University of Geosciences \\ Wuhan, China \\ e-mail: songhengli@163.com
}

\author{
Haobin Dong \\ College of Automation \\ China University of Geosciences \\ Wuhan, China \\ e-mail: donghb@cug.edu.cn
}

\begin{abstract}
A type of remote magnetic field measurement system has been developed to detect magnetic fields simultaneously in three orthogonal directions. The 3-axis magnetic sensor is composed of three orthogonal magnetoresistors. A STM32 operates as the microprocessor for digital signal processing and peripheral module controlling. A GPRS module is connected to the microprocessor to operate as an embedded server. The corresponding client is a mobile smart phone which runs as a remote monitor developed by the Android platform. Some detail of designe and core codes are provided. The test results comes to the conclusion that the precision of the system is sufficient in most magnetic measurement situations. The whole system holds the advangage of ynchronization, remote and portability.
\end{abstract}

Keywords-remote measurement; magnetic field; threedimension; synchronous measurement; Andrid smart phone

\section{INTRODUCTION}

Nowadays, the measurement of magnetic field plays an increasing crucial role in many fields such as scientific research, national defense, industrial production, daily life and so on[1]. Among all those fields, the technology of weak geomagnetic field measurement is performed in various vital situations which are discussed in recent researches[2]. Reference[3] designed a sensor with the magnetic circuit containing set of permanent magnets with various magnetic field values. The redesigned sensor may be referred as a passive eddy-current sensor. Reference[4] focused on three-dimensional nuclear magnetic resonance in structural elucidations of complex samples to obtain high-resolution 3D spectra in inhomogeneous fields. Reference[5] proposed a method for measuring the submarine's induced magnetic field to achieve the goal of fast and long distance measuring. Reference[6] got a precise measurement of a magnetically inhomogeneous sample on a modified a spinner.

All the measurement systems of geomagnetic field mentioned in above references need upgrading on two mainly aspects. The most significant is that geomagnetic field has to be detected simultaneously in three perpendicular directions which are vector decompositions of the measured geomagnetic field. Furthermore, in case of complicated circumstances, it is impossible to lay out a long distance wire to transmission the measured values to the monitor[7]. Recently, the increasing requirements for data acquisition at any time, from any place, regardless the type of remote device or planned operation, has paved the way towards the new technological revolution $[8,9]$.

Therefore, long distance remote monitors and portable devices have to be imported in the system. The advancement and dominance of Android devices in smart phone provides a realizable solution of remote measurement[10].

This paper concentrates on the above two aspects and offers a system which measures three direction magnetic fields simultaneously and transmission the data via wireless network to a smart phone which runs as a monitor. The MCU, STM32F103 controls the DAQ circuit and the communication module to operate as an embedded server. The three directional magnetic fields are sampled by the DAQ simultaneously. The converted data from DAC is calculated to the value of magnetic field which is sent to the smart phone of user via the Wireless Internet. The android-based phone runs software which is developed by Java draw the three curves to provide a friendly GUI.

\section{HARDWARE DESIGN}

\section{A. Integrated framework}

The integrated framework of the system is as shown in Fig .1. The whole hardware involves three parts which are the sensor, the DAQ circuit and the communication module.

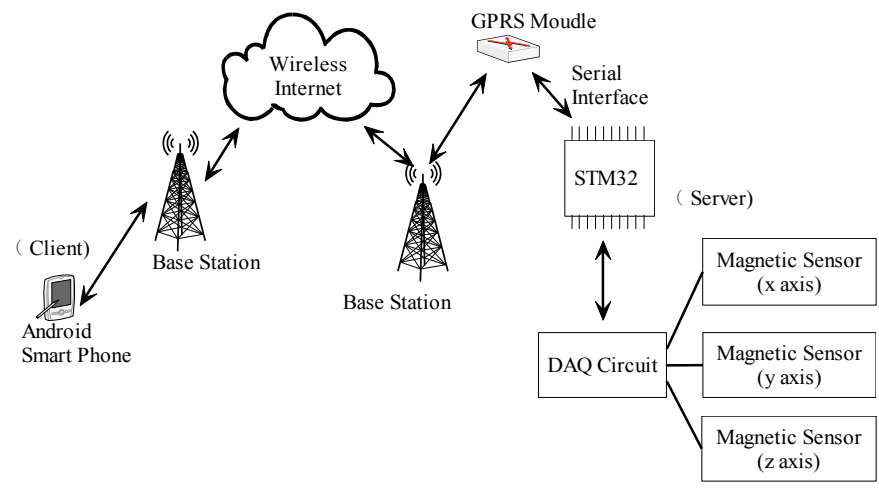

Figure 1. System framework

\section{B. The 3-axis Magnetic Sensor}

The remote measurement system detects the magnetic field by a 3-axis magnetic sensor which is made of three magnetoresistors. These magnetoresistors are fixed 
orthogonally on a DIP footprint module thus the directions of magnetic induction are accordingly orthogonal, as is shown in Fig .2.

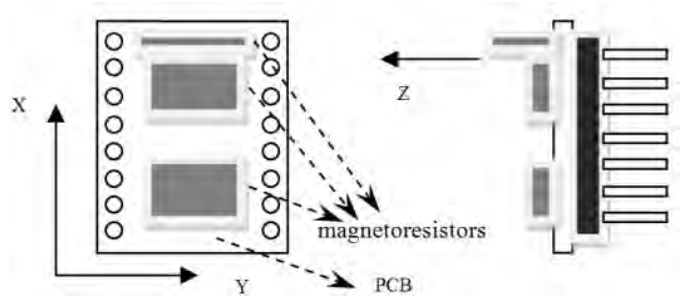

Figure 2. Mechanical installation of the magnetoresistors

Every signal magnetoresistor equivalents a Wheatstone bridge constituted by four permalloy belts inside the sensor. The permalloy with anisotropic magnetoresistance effect leads to the change of sensor output voltage once the value of resistances of the bridge change accordingly. The sensor holds two aluminum circulating current straps, one of which is the set/reset strap with function of sensitivity correction. The other is the offset strap which can be used to oppose the circumstance magnetic field.

The principle of the magnetoresistor is shown in Fig .3. When the magnetoresistor is a zero-magnetic field the resistances of the bridge are all equals to $R$. The $V_{b}$ is the working source of the bridge driving a circuit in the sensor. As an extra magnetic field $M$ applied, magnetization of the permalloy belts changes. The magnetization direction turns to the direction of the current. Consequently, the two opposite resistances of the bridge reduce by $\Delta R$, while the other two opposite resistances increase by $\Delta R$ because of the magnetization's opposite direction to the current.

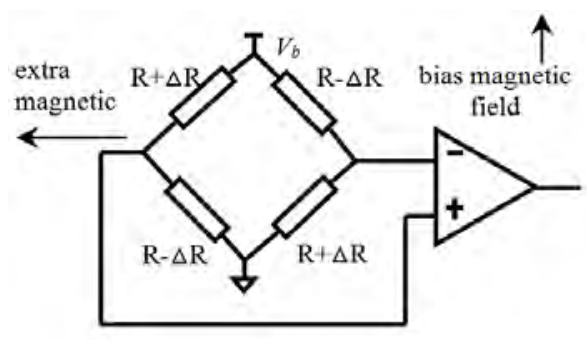

Figure 3. Equivalent circuit of the magnetoresistors

The output voltage is in direct proportion to the value of measured extra magnetic field.

$$
\Delta V_{\text {out }}=\left(\frac{\Delta R}{R}\right) V_{b}
$$

Where the $\Delta R$ is the reduced or increased value of resistances. The $R$ is the equivalent resistance of the Wheatstone bridge's single-arm. The $V_{b}$ is the working source of the bridge.

According to the principle of the magnetoresistor, the output voltage can be also described as follow.

$$
\Delta V_{\text {out }}=V_{b} \cdot M \cdot S
$$

Where the $\mathrm{S}$ is the sensitivity of the magnetoresistor, the $M$ is the value of measured extra magnetic field.

Therefore, we can calculate the value of the extra magnetic field as follow.

$$
M=\Delta V_{\text {out }} /\left(V_{b} \cdot S\right)
$$

Thus, the system need measuring the output voltage to get the value of the magnetic field.

\section{The DAQ Circuit}

The DAQ(Data Acquisition) circuit is consist of a MCU STM32F103 and an AD converter AD7193, as shown in Fig .4.
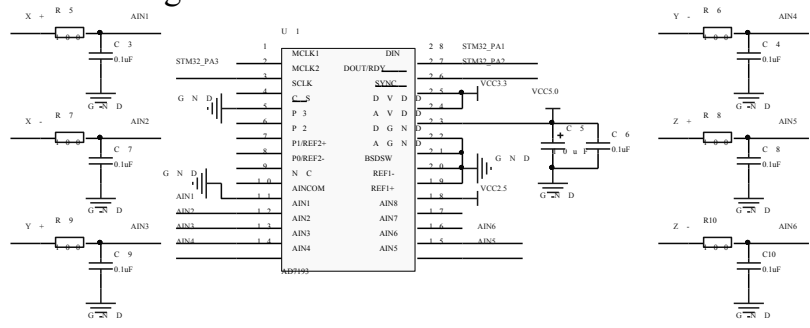

Figure 4. DAQ circuit

The measured three vector components of the magnetic field are led to three sample channels of the AD7193. The analog power source of the $\mathrm{AD}$ shares a same $+5 \mathrm{~V}$ with the sensor, while the digital power source of the $\mathrm{AD}$ is given by the $+3.3 \mathrm{~V}$ of the MCU to avoid additional level translator in the same digital circuit.

For the purpose of increasing SNR, the weak measured voltage signal must be amplified and filtered. These signal process circuit can be simply programed inside the AD7193 which is embedded by a pre-amplifier, a filter and a PGA. The amplification factor can be programed to 1,8 , $16,32,64$, or 128 as needed. The embedded filter shows an excellent noise performance even in a whole data transmission rate. Moreover, AD7193 offers a programmable $50 \mathrm{~Hz} / 60 \mathrm{~Hz}$ notch filter.

\section{The Voltage Reference}

An extra voltage reference is designed to connect to AD7193. The reference IC is selected to be LTC6655-2.5. This IC has ultra-low working noise $\left(625 \mathrm{nV}_{\mathrm{p}-\mathrm{p}}\right)$ in the frequent band from $0.1 \mathrm{~Hz}$ to $10 \mathrm{~Hz}$. In the DAQ circuit, the power source pin of LTC6655-2.5 is connected to the power source pin of the sensor. Therefore the low frequency noise on power line is minimized. Fig .5 shows the voltage reference circuit where $\mathrm{C} 13 \sim \mathrm{C} 16$ are decoupling capacitors

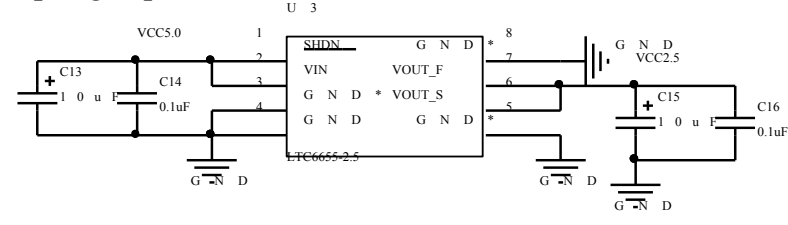

Figure 5. Voltage reference circuit

\section{E. The GPRS Module}

In order to realize the remote measurement an industrial grade GPRS module operates as a network server. The MCU calculates the magnetic field according to the equal (3) base on the sampled voltage. As getting the result, the MCU send the data via the serial interface to the GPRS module.

The connection between GPRS module and MCU is as shown in Fig .6. 
The GPRS module is based on the SIM900A which is programed by AT orders through pins of the MCU. The key AT orders to initialize and construct a server is as shown in Tab. I.

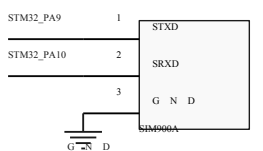

Figure 6. Connection between GPRS module and MCU

TABLE I. AT ORDERS TO ESTABLISH SERVER

\begin{tabular}{c|c|c}
\hline Step & AT orders & Implication \\
\hline 1 & AT+CGCLASS=B & $\begin{array}{c}\text { To set mobile class supporting } \\
\text { data switch }\end{array}$ \\
2 & AT+CGDCONT=1 & $\begin{array}{c}\text { To set PDP context and internet } \\
\text { protocol }\end{array}$ \\
3 & AT+CDDCONT=1 & To apply for GPRS service \\
5 & AT+CIPCSGP=1 & To set GPRS connection model \\
6 & AT+CIPHEAD=1 & To set receiving data IP header \\
7 & AT+CLPORT=TCP & To get TCP protocol ready \\
\hline
\end{tabular}

\section{SOFTWARE DESIGN}

\section{A. Function Design and Operation Procedure}

The long distance monitor is deployed on a smart mobile phone with the Android OS. The smart phone which is developed by Java language operates as the client. This solution is optimized. It has friendly and convenient GUI. The cost of development can be deduced because of no additional hardware needed. The data communication is realizable and reliable due to the large coverage of the Wireless Mobile Internet.

The function of client is designed as follow.

1) Log in: To obtain the IP address of the server and connect to the server in socket model.

2) Receive user's order and upload: To obtain and the user's operation order through touch panel, code the order and upload to the server.

3) Acquisition and display: To receive the data from the server, decode the data and display the measured value on the panel.

The operation procedure is designed according to the function and is shown in Fig .7.

The client connects to the server according to the latest IP address. If the connection fails, it is implied that the dynamic IP address has been modified. The client sends an acquiring SMS via GSM to the server, waits for the new IP address sent from the server in a special string formula and then reconnects to the server to establish a socket handshaking. The orders from the user are coded and sent to the server via GPRS wireless network communication. After that, the client waits for response from the server and gets ready to receive the data.

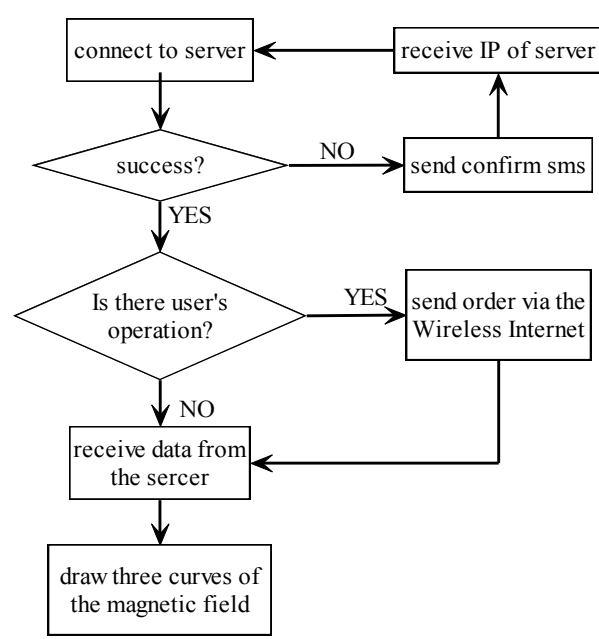

Figure 7. Operation procedure of the client

\section{B. Main Software Moudel and Core Codes}

1) Layout: According to the above function design, the UI of the client involves several widgets such as TextView, Button, Swich and so on. The codes of layout are saved in the file Layout.xml. The RelativeLayout is chosen. The main widgets and their functions are shown in Tab.2.

TABLE II. VITAL WIDGETS IN LAYOUT

\begin{tabular}{c|c|c}
\hline Name & Category & Function \\
\hline Button01 & Button & $\begin{array}{c}\text { To connect to server } \\
\text { To obtain dynamic IP }\end{array}$ \\
Button02 & Button & Address \\
Textview01 04 & TextView & To display operation tips \\
SurfaceView01 03 & SurfaceView & To draw three curves \\
\hline
\end{tabular}

2) Swith Listener: The switch lisener is used to accept the user's order and display the status of connection. The core codes of setting listener is as follow.

mySwitch.setOnCheckedChangeListener(new

OnCheckedChangeListener()

where mySwitch is a new Switch class object. Its metheod is as follow:

class Switch Listener implements

OnCheckedChangeListener()\{

String msg;

if(mySwitch.isChecked())//Switch clicked msg = myEditText.getText().toString(); /*generate order string*/

else //Switch unclicked msg $=$ myEditText.getText().toString(); $/ *_{\text {generate order string*/ }}^{*}$

try $\{/ /$ establish connection thread System.out.println("out: " + msg); /*send message to the server*/

_ $\quad$ printWriter.print $(\mathrm{msg})$ printWriter.flush(); \}catch (Exception e) \{\}$\}$

3) Connect to the server in Socket model: The socket communication offers direct data transmission after 
established without any request for send. The connetion program is implemented by a Button listener. The method onClick() is set to respond the click of the Button. The core codes of establish of socket connetion is as follow:

private static final String SERVERIP ;

private static final int SERVERPORT $=5000$;

private InetSocketAddress isa = null;

private Socket_socket $=$ null;

private int timeout $=10000$;

public void onClick(View v) \{

Toast.makeText(TCP1Activity.this, "Connect to TCP server", Toast.LENGTH_LONG).show(); try \{

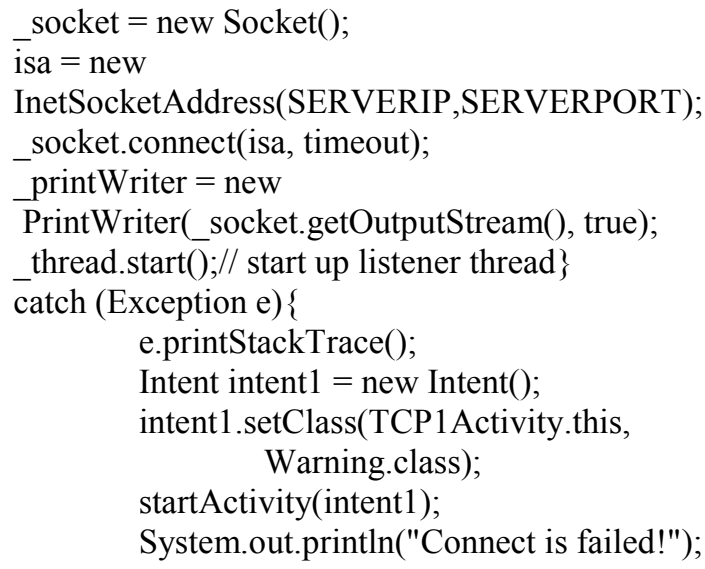

\section{SYSTEM TEST}

The schematic design and PCB layout design have been accomplished. The hardware and software debugging have been also performed to make the whole system normal. The PCB of the sensor and the main control board is shown in Fig .8.

To test the feasibility and the stability of the system, the output voltages of the sensor was measured. As is shown in Tab.III, the voltages of $\mathrm{X}, \mathrm{Y}$, and $\mathrm{Z}$ axis were repeatedly measured.

TABLE III. OUtPut Voltages of THREe AXES

\begin{tabular}{c|c|c}
\hline $\begin{array}{c}\text { Output of X-axis } \\
(\mathbf{m V})\end{array}$ & $\begin{array}{c}\text { Output of Y-axis } \\
(\mathbf{m V})\end{array}$ & $\begin{array}{c}\text { Output of Z-axis } \\
(\mathbf{m V})\end{array}$ \\
\hline-7.89828 & -6.63404 & -0.9383 \\
-7.89814 & -6.62886 & -0.93814 \\
-7.89723 & -6.61591 & -0.93509 \\
-7.89721 & -6.61542 & -0.93494 \\
-7.89809 & -6.61595 & -0.97355 \\
-7.89543 & -6.61462 & -0.99222 \\
-7.89817 & -6.61423 & -0.99989 \\
-7.89775 & -6.61573 & -1.00051 \\
-7.89778 & -6.61476 & -0.98007 \\
-7.89535 & -6.61368 & -0.98248 \\
\hline
\end{tabular}

From the measured data listed in Tab. III, it is illustrated that the maximum absolute error are respectively $2.0 \mathrm{nV}, 15.7 \mathrm{nV}$ and $32.5 \mathrm{nV}$. The reason of larger error on z-axis is analyzed to be the asymmetry of three sample channels.

According the error of output voltage, the maximum measurement error of the system is $100 \mathrm{nT}$.

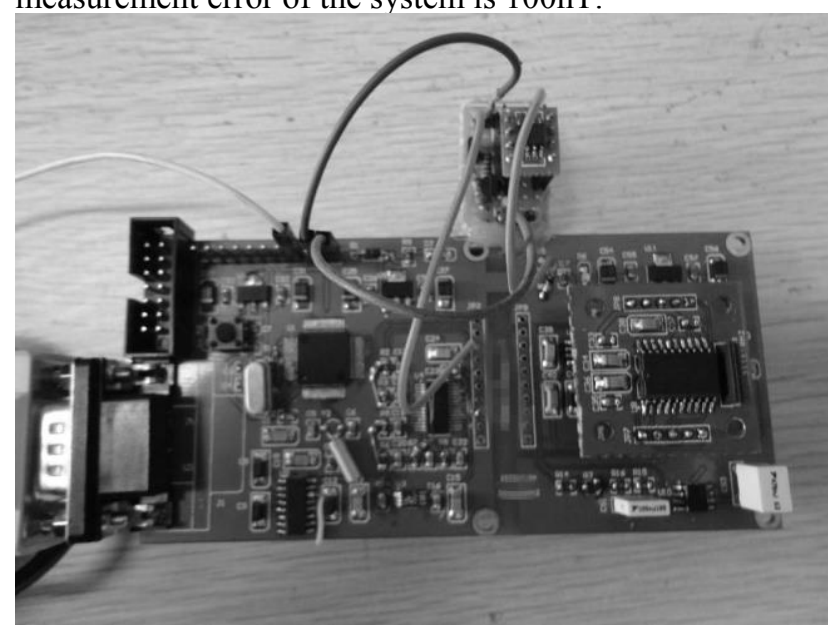

Figure 8 . Photo of the system

The whole system was tested in an outdoor space. The three dimensional magnetic field was sampled per second. The MCU working as server sent the data to a mobile phone via the Wireless Internet.

The operation interface and the three curves are shown in Fig .9. The horizontal axis of the curve is sample time from 0 to 160 seconds. While the vertical axis of the curve is the value of magnetic field with the unit of nT.

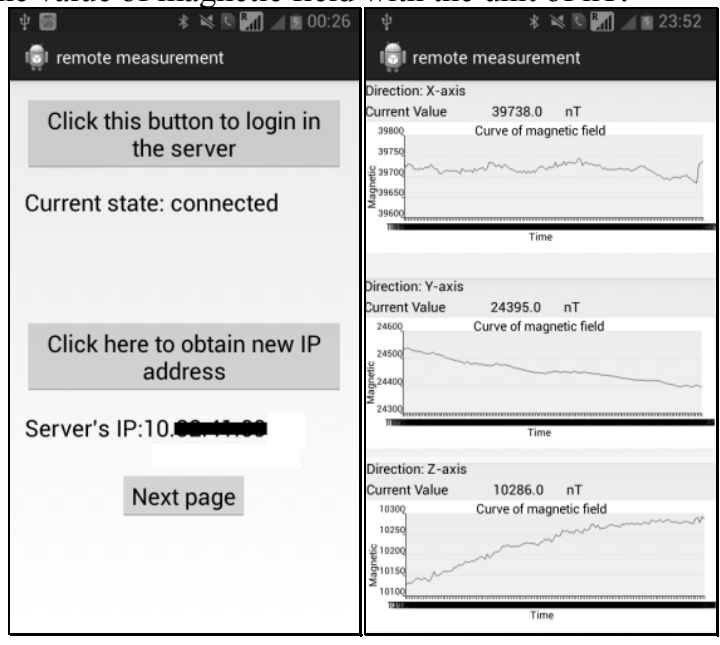

Figure 9. Printed screens of the running smart phone

\section{CONCLUSION}

From the above design and test of the remote measurement system, following conclusions can be drawn definitely.

1) The precision of the system is $100 \mathrm{nT}$, which is sufficient in most magnetic measurement cases.

2) The system offers a method of synchronous measurement which can be widely used in magnetic detections. 
3) The system is remarkable portable and usable due to the technology of smart phone development based on Android OS.

4) The distance of measurement is unlimited thanks for the wide spread mobile network.

5) The system has a broad application prospect because of its synchronization, remote and portability.

\section{ACKNOWLEDGMENT}

This work is proudly supported by the National Natural Science Foundation of China (NO. 41474158).

\section{REFERENCES}

[1] X. Zhang, J. Liu, M. Li, and Y. Fu, "Remote measurement of magnetic field distribution in the solenoid," Proc. Computational Materials Science(CMS 2011), Trans Tech Publications, 2011, pp.1591-1594, doi:10.4028/www.scientific.net/AMR.268-270.159

[2] X. Wang and K.V. Kamenev, "Review of modern instrumentation for magnetic measurements at high pressure and low temperature," Fizika Nizkikh Temperatur, vol. 40, no. 8, 2014, pp.946-958.

[3] R. Przysowa, E. Rokicki and P. Majewski, "Optimized magnetic sensors to measure speed and position in adverse environments," Proc. IET and ISA 60th International Instrumentation Symposium 2014, Institution of Engineering and Technology, 2014, doi:10.1049/cp.2014.0552.

[4] Y. Huang, Z. Zhang, K. Wang, S. Cai, and Z. Chen, "HRJCOSY: A three-dimensional NMR method for measuring complex samples in inhomogeneous magnetic fields," Chem Phys Lett, vol. 609 , 2014, pp.21-25, doi:10.1016/j.cplett.2014.06.025.

[5] Z. Zhang and J. Yi, "Measuring the submarine's induced magnetic field by geomagnetic simulation method," Proc. Electronic Engineering and Information Science 2014, Trans Tech Publications Ltd, 2014, pp.579-584, doi:10.4028/www.scientific. net/AMR.981.579.

[6] P. Marton, "The principles of the complete measurement of an offcentred elementary magnet on a spinner magnetometer with a coil sensor," J Magn Magn Mater, vol. 372, 2014, pp.236-246, doi:10.1016/j.jmmm.2014.07.047.

[7] H. Qin, J. Hu and L. Zheng, "Design of remote weak magnetic field measurement system," Proc. International Conference on Mechatronic and mbedded Systems and Applications( MESA 2007), Institute of Electrical and Electronics Engineers Inc., 2007, doi:10.1109/MESA.2006.296984.

[8] J. Nadvornik and P. Smutny, "Remote control robot using Android mobile device," Proc. Proceedings of the 2014 15th International Carpathian Control Conference(ICCC 2014), IEEE Computer Society, 2014, pp.373-378, doi:10.1109/CarpathianCC. 2014.6843630.

[9] N. Truong and D. Vu, "Remote monitoring and control of industrial process via wireless network and Android platform," Proc. 2012 International Conference on Control, Automation and Information Sciences(ICCAIS 2012), IEEE Computer Society, 2012, pp.340-343, doi:10.1109/ICCAIS.2012.6466614.

[10] [P. Pierleoni, L. Pernini, A. Belli, and L. Palma, "An android-based heart monitoring system for the elderly and for patients with heart disease," International Journal of Telemedicine and Applications, vol. 2014, 2014, doi:10.1155/2014/625156. 